\title{
EDITORIAL
}

\section{LA REVISTA ESPAÑOLA DE SALUD PÚBLICA INGRESA EN EL SOCIAL SCIENCE CITATION INDEX DE THOMSON SCIENTIFIC}

Cristina Pérez Andrés (1), Emilio Delgado López-Cózar (2) y Evaristo Jiménez Contreras (2)

(1) Comité de Redacción de la Revista Española de Salud Pública. Ministerio de Sanidad y Consumo.

(2) EC3: Evaluación de la Ciencia y de la Comunicación Científica. Departamento de Biblioteconomía y Documentación. Universidad de Granada.

Thomson es una empresa dedicada a proporcionar información especializada en diferentes áreas: financiera, cuidados de salud, educación, legal, científica e impuestos y contabilidad ${ }^{1}$. En el caso de la información científica la gestión se hace a través de Thomson Scientific, antes Thomson ISI.

Thomson Scientific elabora información especializada que comercializa en una serie de bases de datos: Science Citation Index (SCI), Social Science Citation Index (SSCI) y Arts and Humanities Citation Index (A\&HCI), actualmente reunidas en el Web of Science (WOS) $)^{2}$, las cuales se han convertido en los últimos años en el referente mundial a la hora de identificar la llamada ciencia internacional y, por extensión, la investigación de mayor calidad e impacto. Debido al carácter selectivo y a las prestaciones bibliométricas que estas bases de datos ofrecen, gobiernos e instituciones han convertido al Institute for Scientific Information (ISI) en la fuente de datos habitual para las diferentes actividades evaluadoras. Entre ellos cabe destacar el factor de impacto, el cual es el producto más conocido habitualmente por los autores y lectores de artículos científicos.
Por estas razones, y por otras que desgranaremos posteriormente, estar presente en las bases de datos del ISI ha llegado a ser una prioridad para investigadores, instituciones y revistas. El problema es que se trata de un anhelo compartido por miles de revistas en el mundo. Muchas son las que lo solicitan (más de 2.000 cada año) y pocas las que lo consiguen. Este año una de ellas ha sido la Revista Española de Salud Pública (RESP). Que una revista científica española como la RESP ingrese en el ISI posee una significación especial que debe ser resaltada y fuente de satisfacción no sólo para los editores de la revista sino para toda la comunidad científica y profesional a la que representa.

En primer lugar conviene resaltar que en las bases de datos del ISI (http://scientific. thomson.com/mjl) sólo figuran 9.000 revistas científicas de las más de 100.000 que circulan en todo el mundo. Así el SCI, cuya cobertura arranca de 1945, tiene indizadas en la actualidad 6.543 revistas distribuidas en 71 categorías; el SSCI (desde 1956) posee 1.917 revistas distribuidas en 54 categorías y el A\&HCI (desde 1975) indiza 1.149 revistas distribuidas en 27 categorías $^{2}$. Pues bien, la RESP pasa a ser una de las 67 revistas 
españolas que han superado el proceso de evaluación (tabla 1), tras solicitar su ingreso en agosto de 2001, y hoy ya se encuentra indizada en el ISI. De ellas 36 figuran en el SCI, 13 en el SSCI y 18 en el A\&HCI. Estos datos adquieren su verdadero valor si reparamos en el hecho de que la RESP se convierte en la primera revista española sobre salud pública que accede a las bases de datos del ISI y en una de las pocas revistas iberoamericanas que de esa misma disciplina lo han conseguido (Salud Pública de México y Revista de Saude Publica).

En segundo lugar cabe señalar que el ISI es muy selectivo debido a las exigencias que impone y que deben cumplir las revistas que ingresan en él, exigencias que finalmente repercuten beneficiosamente en las revistas que indiza, tanto en lo que concierne al aumento de su visibilidad y prestigio como en lo que se refiere a la mejora de sus prácticas editoriales internas. Desde hace años el ISI cuenta con un riguroso proceso de evaluación de las revistas candidatas a ser indizadas en sus fondos ${ }^{3}$. Los criterios de selección de revistas empleados por el ISI se pueden estructurar en cuatro grandes apartados:

1. Cumplimiento de los estándares de publicación en revistas científicas.

2. Cobertura temática de la revista.

3. Representatividad internacional.

\section{Repercusión y visibilidad científica.}

El primero de ellos, que actúa como prerrequisito para el resto, es el cumplimiento de los estándares internacionales de publicación científica, entre los que cabe subrayar la regularidad y puntualidad, la calidad y corrección de los títulos de los artículos, en el sentido de que sea descriptivos y ajustados a los contenidos de los trabajos; la calidad de los resúmenes y palabras clave, que debe entenderse en el mismo sentido que el punto anterior; la indicación de las instituciones (todas) a las que pertenecen los autores firmantes; la corrección y exhaustividad de las referencias bibliográficas, y la práctica acreditada y regular del sistema de evaluación por pares (externo y anónimo) de los originales que se envían a la revista.

El segundo aspecto que se valora es la cobertura temática de la revista. En este caso el ISI pondera que la revista ocupe un espacio científico diferenciado que sea de interés para la base de datos, bien porque se trate de un campo emergente e innovador o porque la revista represente a una comunidad sólida cuyas aportaciones son científicamente relevantes. Aspecto éste que representa una «ventana de oportunidad» para las revistas españolas, tradicionalmente maltratadas en esta base de datos, sesgo que el ISI está tratando de corregir.

El tercer aspecto tiene que ver con la representatividad internacional de la revista. En este punto el ISI exige que la revista esté patrocinada por una organización solvente, que su equipo editorial (especialmente el comité científico) lo integren investigadores de prestigio, lo que es extensible a los científicos que publican en ella e, incluso a la audiencia a la que se dirige, en el sentido de que sea internacional y no meramente local, lo que es observable a través de su presencia en bibliotecas y, muy especialmente, en bases de datos.

Y, por último, pero no menos importante, evalúa la repercusión científica de la revista a través del recuento de las citas que recibe. El hecho de que una revista no esté incluida en el ISI no significa que su impacto medido en citas no pueda ser estudiado. Ello se puede hacer de forma directa, contabilizando las citas que los artículos que publica la misma reciben en el WOS o de forma indirecta, haciendo un seguimiento del impacto científico de los autores que constituyen el comité editorial de la revista o de los autores que publican en ella, en ambos casos se puede calcular el impacto que alcanzan las publica- 
ciones internacionales (léase la publicadas en revistas ISI) de las que son responsables. Precisamente este riguroso filtro, entre otros factores, es el que dota de exclusividad a estas bases de datos y otorga reputación a las revistas que logran franquearlo, como es el caso de la RESP.

Por ofrecer algunos datos generales de los procesos de evaluación de Thomson Scientific, señalaremos que aproximadamente unas 2.000 revistas son evaluadas cada año. Se incluyen aquí tanto las nuevas que se someten a valoración para su posible inclusión o cobertura como las que se reevalúan para su posible salida o cambio de ubicación en los campos en que se organiza la base de datos. Finalmente, cada año son incluidas entre 150-200 nuevas revistas.

Como consecuencia de todo ello no es exagerado afirmar que en las bases de datos ISI se encuentra la bibliografía científica de mayor difusión, visibilidad e impacto internacional. Pero lo que realmente ha transmutado a las bases de datos ISI en «oscuro objeto de deseo» es la posibilidad que ofrece de conocer el impacto y repercusión científica que tienen los trabajos publicados a partir del recuento de las citas bibliográficas que reciben. Con el paso del tiempo los indicadores bibliométricos que proporcionan han devenido en herramientas imprescindibles para la evaluación del rendimiento investigador de las instituciones, de las revistas y de los propios científicos. Publicar en una revista indizada por el ISI se ha convertido en un signo intrínseco de calidad valorado positivamente por los más diversos sistemas de evaluación. En el caso de España, tanto la Comisión Nacional Evaluadora de la Actividad Investigadora (CNEAI $)^{4}$ desde 1990, como la Agencia Nacional de Evaluación de la Calidad y Acreditación (ANECA) ${ }^{5}$ desde 2002, organismos encargados de evaluar y acreditar el rendimiento científico de los investigadores, valoran preferentemente las aportaciones que sean artículos en revistas de prestigio reconoci- do, aceptándose como tales las que ocupen posiciones relevantes en los listados por ámbitos científicos en el «Subject Category Listing» del Journal Citation Reports del Science Citation Index (SCI), del Social Sciences Citation Index (SSCI) y del Arts and Humanities Citation Index (AHCI) (Institute of Scientific Information, -ISI-Philadelphia, PA, USA).

Estas prácticas, que no son privativas de nuestro país y están extendidas por todo el planeta, han provocado que las revistas cubiertas por el ISI atraigan los mejores trabajos de investigación, aquéllos que poseen la mayor relevancia científica, tanto por el objeto de investigación como por su calidad metodológica. De ahí la trascendencia que para la vida de una revista tiene ingresar en las bases de datos del ISI.

Para entender mejor el contexto en el que se produce el ingreso de la RESP hay que aludir a dos factores externos que lo han propiciado. De un lado la política de Thomson Scientific de ampliar significativamente su fondo editorial. Consciente de sus sesgos geográficos (predominio de las revistas anglosajonas y en lengua inglesa) así como de sus lagunas en la cobertura de revistas de Ciencias Sociales y de orientación aplicada, ha decidido solventar este talón de Aquiles incluyendo unas 400 revistas. En última instancia explica todos estos movimientos la competencia de productos como Scopus (http://www.info.scopus.com), Google Scholar (http://scholar.google.es), Psychinfo (http://www.apa.org/psycinfo), e incluso Índice de Impacto de las Revistas Españolas de Ciencias Sociales (IN-RECS: http://ec3. ugr.es/in-recs), que han roto el monopolio de las bases de datos ISI en cuanto al tratamiento, búsqueda y recuperación a través las citas bibliográficas, aunque no de los indicadores bibliométricos donde ISI sigue siendo el referente.

De otro lado, la incorporación de revistas españolas a las bases de datos del ISI, que ha 
sido un goteo continuado en los últimos años, es en última instancia reflejo del creciente peso de la ciencia española en el ámbito internacional, que en el último año ha experimentado una importante aceleración fruto, al menos en una parte no desdeñable, de la actividad desplegada por la Fundación Española para la Ciencia y la Tecnología (FECYT) (http://www.fecyt.es), interlocutor nacional frente a la empresa norteamericana. Esta actividad se ha concretado en la incorporación de varias revistas españolas a la sección de Ciencias Sociales, la más «descuidada» de la base de datos hasta ahora en lo que a la cobertura se refiere, ya que sólo recogía dos títulos: Psicothema y European Journal of Psychiatry. A partir de 2006 a esta lista se han unido otras 11 revistas, entre las que se cuenta la RESP, (tabla 1), que a final de año llegarán a ser 20. Por otra parte desde la FECYT también se están tomando medidas para apoyar a las revistas científicas españolas en su necesario proceso de apertura al exterior (léase de nuevo su incorporación a los listados de las bases de datos internacionales, especialmente las del ISI). En un futuro más o menos inmediato, y si se concretan las medidas que están discutiéndose, habrá un apoyo tangible y el reconocimiento consiguiente a las revistas que incrementen su visibilidad internacional.

Por último, cabe comentar que la RESP ha sido incluida dentro del SSCI en la categoría Public, Environmental and Occupational Health, una categoría compuesta por 70 revistas (tabla 2) que, de acuerdo con la definición del ISI, cubre «materias sobre medicina social, hábitos saludables, educación para la salud, seguridad y salud mental y comunitaria. En esta categoría se incluyen también recursos relacionados con la salud de grupos específicos tales como adolescentes, personas mayores, o mujeres». Esta adscripción hay que calificarla al menos como discutible, pues esta misma categoría figura en el SCI (tabla 2) cubriendo un ámbito temático más acorde con los contenidos de la RESP, ya que incluye recursos relacionados con la epidemiología, higiene, y salud; enfermedades parasitarias y parasitología, medicina tropical, medina industrial, salud laboral, control de infecciones y medicina preventiva. También incluye recursos sobre salud y medioambiente, etiología y control del cáncer; medicina de la aviación, medicina de los aerosoles y medicina». Es necesario destacar que éste es el único caso en el que una misma categoría figura en los dos índices, algo que se ha dado históricamente en muy contadas ocasiones, y que habla a las claras de una cierta indefinición respecto a las fronteras de la especialidad, seguramente por el carácter transversal que posee. De hecho hay 19 revistas que figuran en ambas categorías y por lo tanto en los dos índices (tabla 2).

Se trata de una categoría dominada absolutamente por las revistas anglosajonas (tabla 3) que llegan a representar más del 90\% de las publicaciones, y particularmente, por las revistas de Estados.Unidos e Inglaterra cuyos valores agregados superan el $85 \%$. Esta distribución pone de nuevo de manifiesto los sesgos geográficos y lingüísticos de los que adolecen las bases de datos del ISI. El (80\%) de las revistas están editadas por editoriales comerciales (tabla 4), ocupando un papel destacado las multinacionales de la edición científica (Elsevier, Blackwell, Kluwer...). No existe en esta categoría, a diferencia de otras, un monopolio tan acusado por parte de estas editoriales.

Conviene tener en cuenta estos datos para hacer una correcta lectura e interpretación del futuro factor de impacto de la RESP, el cual no aparecerá hasta 2008 porque, como es bien sabido, la fórmula de este famoso indicador se construye con las citas y artículos publicados en un período de dos años, que en el caso de las revistas ahora aceptadas serán 2006 y 2007. Es oportuno recordar que por ser un indicador ampliamente utilizado en la evaluación de los científicos, el factor de impacto de una revista ha sido objeto de investigación y de innumerables discusiones ${ }^{6-9}$. Por tanto, existe abundante literatura 
Tabla 1

Revistas españolas indizadas por Thomson Scientific

\begin{tabular}{|c|c|c|c|}
\hline \multirow{2}{*}{ 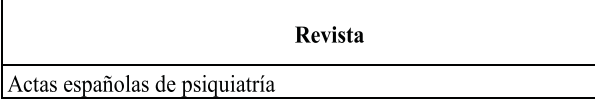 } & \multirow{2}{*}{$\begin{array}{c}\text { FI } \\
0,286\end{array}$} & \multicolumn{2}{|c|}{$\begin{array}{c}\text { Categoría en la que se encuentra y FI } \\
\text { agregado de la misma }\end{array}$} \\
\hline & & Neurociencias & 3,552 \\
\hline \multicolumn{4}{|l|}{ AIDS Review } \\
\hline Archivos de bronconeumología & 1,401 & Sistema respiratorio & 2,99 \\
\hline Nefrología & 0,466 & Urology \& nephrology & 2,697 \\
\hline Medicina clínica & 1,074 & Medicine, general \& internal & 4,326 \\
\hline Revista clínica española & 0,273 & Medicine, general \& internal & 4,326 \\
\hline Revista española de cardiología & 1,769 & Cardiac \& cardiovascular systems & 3,603 \\
\hline Revista española de enfermedades digestivas & 0,535 & Gastroenterology \& hepatology & 3,332 \\
\hline Neurología & 0,571 & Clinical neurology & 2,539 \\
\hline Revista de neurología & 0,391 & Clinical neurology & 2,359 \\
\hline \multirow[t]{2}{*}{ Neurocirugía } & \multirow[t]{2}{*}{0,232} & Neurosciences & 3,552 \\
\hline & & Surgery & 1,741 \\
\hline Drug news \& perspectives & 2,159 & Pharmacology \& pharmacy & 2,604 \\
\hline Drugs of today & 1,248 & Pharmacology \& pharmacy & 2,604 \\
\hline Methods and findings in experimental and clinical pharmacology & 0,798 & Pharmacology \& pharmacy & 2,604 \\
\hline Drugs of the future & 0,547 & Pharmacology \& pharmacy & 2,604 \\
\hline Histology and histopathology & 2,023 & Cell biology & 5,551 \\
\hline Journal of physiology and biochemistry & 0,934 & Biochemistry \& molecular biology & 4,374 \\
\hline \multirow[t]{2}{*}{ Enfermedades infecciosas y microbiología clínica } & \multirow[t]{2}{*}{0,905} & Infectious diseases & 3,435 \\
\hline & & Microbiology & 3,254 \\
\hline \multirow[t]{3}{*}{ International microbiology } & \multirow{3}{*}{1,868} & Biotechnology \& applied microbiology & 2,795 \\
\hline & & Microbiology & 3,254 \\
\hline & & Physiology & 3,085 \\
\hline \multirow[t]{3}{*}{ Grasas y aceites } & \multirow{3}{*}{0,194} & Química aplicada & 1,557 \\
\hline & & Ciencia y tecnología de los alimentos & 1,346 \\
\hline & & Pathology & 2,326 \\
\hline International journal of developmental biology & 2,051 & Developmental biology & 5,098 \\
\hline \multirow[t]{2}{*}{ Journal of Investigational Allergology and Clinical Immunology } & \multirow[t]{2}{*}{0,857} & Allergy & 3,213 \\
\hline & & Inmunollogy & 4,181 \\
\hline Afinidad & 0,220 & Química & 2,985 \\
\hline \multirow[t]{3}{*}{ Archives of computational methods in engineering } & \multirow{3}{*}{1,400} & Computer science, interdisciplinary applications & 1,577 \\
\hline & & Engineering, multidisciplinary & 0,862 \\
\hline & & Mathematics, interdisciplinary applications & 1,636 \\
\hline Ardeola & 0,509 & Ornitología & 0,906 \\
\hline Boletín de la sociedad española de cerámica y vidrio & 0,684 & Materials science, ceramics & 0,711 \\
\hline Collectanea Mathematica & & Mathematics & 0,501 \\
\hline \multirow[t]{2}{*}{ Materiales de construcción } & \multirow[t]{2}{*}{0,542} & Construction \& building technology & 0,504 \\
\hline & & Materials science, multidisciplinary & 1,643 \\
\hline Publications matematiques & 0,659 & Mathematics & 0,501 \\
\hline Revista de metalurgia & 0,414 & Metallurgy \& metallurgical engineering & 0,939 \\
\hline Revista matemática iberoamericana & 0,855 & Mathematics & 0,501 \\
\hline Scientia marina & 1,036 & Marine \& freshwater biology & 1,431 \\
\hline Spanish Journal of Agricultural Research & - & Agriculture, Multidisciplinary & 1,366 \\
\hline Test & 1,163 & Statistics \& probability & 1,389 \\
\hline World Rabbit Science & - & Agriculture, Dairy \& Animal Science & 1,046 \\
\hline Boletín de la Asociación de Geógrafos Españoles & - & Geography, & 1,204 \\
\hline El Profesional de la Información & - & Information Science \& Library Science & 0,818 \\
\hline
\end{tabular}


Tabla 1 (Cont.)

Revistas españolas indizadas por Thomson Scientific

\begin{tabular}{|c|c|c|c|}
\hline \multirow{2}{*}{$\begin{array}{l}\text { Revista } \\
\text { European Journal of Psychiatry }\end{array}$} & \multirow{2}{*}{$\frac{\text { FI }}{0,018}$} & \multicolumn{2}{|l|}{$\begin{array}{c}\text { Categoría en la que se encuentra y FI } \\
\text { agregado de la misma }\end{array}$} \\
\hline & & Psychiatry & 1,326 \\
\hline \multirow[t]{2}{*}{ Infancia y Aprendizaje } & \multirow[t]{2}{*}{-} & Psychology, Developmental; & 1,818 \\
\hline & & Psychology, Educational & 1,162 \\
\hline International Journal of Clinical and Health Psychology & - & Psychology, Clinical & 1,925 \\
\hline Investigaciones Económicas & - & Economics & 0,827 \\
\hline Psicológica & - & Psychology, Experimental & 2,040 \\
\hline Psicothema & 0,874 & Psychology, Multidisciplinary & 0,650 \\
\hline Revista de Economía Aplicada & - & Economics & 0,827 \\
\hline Revista Española de Pedagogía & - & Education \& Educational Research & 0,525 \\
\hline Revista Española de Salud Pública & - & Public, Environmental And Occupational Health & 1,663 \\
\hline Scripta Nova-Revista Electrónica de Geografia y Ciencias Sociales & - & Geography & 1,204 \\
\hline The Spanish Journal of Psychology & - & Psychology, Multidisciplinary & 1,472 \\
\hline Al-Qantara & - & History; Religion & \\
\hline Anuario Filosófico & - & Philosophy & \\
\hline Arbor-Ciencia Pensamiento y Cultura & - & Humanities, Multidisciplinary & \\
\hline Archaeofauna & - & Archaeology & \\
\hline Archivo Español de Arte & - & Art & \\
\hline Boletín de la Real Academia Española & - & Literature, Romance & \\
\hline Cuadernos Hispanoamericanos & - & & \\
\hline Goya & - & Art & \\
\hline Hispania-Revista Española de Historia & - & History & \\
\hline Insula-Revista de Letras y Ciencias Humanas & - & Literary Theory \& Criticism & \\
\hline Pensamiento & - & Philosophy & \\
\hline Revista de Dialectología y Tradiciones Populares & - & Folklore & \\
\hline Revista de Filología Española & - & Language \& Linguistics Theory; Literature, Romance & \\
\hline Revista de Indias & - & History & \\
\hline Revista de Literatura & - & Literature, Romance & \\
\hline Revista de Occidente & - & Humanities, Multidisciplinary & \\
\hline Studia Monastica & - & Religion & \\
\hline Sefarad & - & Religion & \\
\hline Theoria-Revista de Teoría Historia y Fundamentos de la Ciencia & - & History \& Philosophy Of Science & \\
\hline
\end{tabular}

y evidencias sobre los factores sociológicos y estadísticos que le afectan y pueden condicionarlo $^{9,10}$. Entre otros, los siguientes son los más importantes:

1. Estadísticamente hablando el FI no es más que un promedio: el número medio de citas recibidas por los artículos publicados en una revista. Constatado el hecho de que la distribución de citas entre los artículos de una revista no se ajusta a una curva normal (es no paramétrica), un pequeño número de artículos reciben altas tasas de citación y, por el contrario, la mayoría de los artículos no son citados o reciben tasas ínfimas de cita- ción, nunca debe tomarse el FI de una revista como representativo de un artículo publicado en ella.

2. El FI de una revista depende directamente de dos grandes variables. Por una parte, del tamaño de la comunidad científica de la que la revista no es más que una expresión (mayor número de científicos, centros de investigación y publicaciones suponen potencialmente un mayor número de citas); y por otra, de la naturaleza de la disciplina o especialidad a la que la revista se adscriba y, especialmente, de los hábitos de publicación y prácticas de citación que imperen en ella. 
Tabla 2

Revistas indizadas en la categoría Public, Environmental and Occupational Health del Social Sciences Citation Index*

\begin{tabular}{|c|c|c|c|c|c|}
\hline & SSCI & SCI & FI & $\begin{array}{l}\text { Total citas } \\
2005\end{array}$ & $\begin{array}{l}\text { Artículos } \\
2005\end{array}$ \\
\hline Annual review of public health & $\mathrm{X}$ & $\mathrm{X}$ & 3,674 & 1628 & 24 \\
\hline American journal of public health & $\mathrm{X}$ & $\mathrm{X}$ & 3,566 & 17954 & 297 \\
\hline Journal of epidemiology and community health & $\mathrm{X}$ & $\mathrm{X}$ & 3,003 & 5197 & 162 \\
\hline Psychiatric services & $\mathrm{X}$ & & 2,700 & 4129 & 173 \\
\hline Social science \& medicine & $\mathrm{X}$ & & 2,619 & 13990 & 460 \\
\hline Nicotine \& tobacco research & $\mathrm{X}$ & $\mathrm{X}$ & 2,549 & 1257 & 87 \\
\hline American journal of health promotion & $\mathrm{X}$ & & 2,495 & 1362 & 45 \\
\hline Tobacco control & $\mathrm{X}$ & $\mathrm{X}$ & 2,415 & 2048 & 82 \\
\hline Sociology of health \& illness & $\mathrm{X}$ & & 2,169 & 1132 & 41 \\
\hline Journal of womens health and gender-based medicine & & & 2,111 & 747 & 0 \\
\hline Journal of adolescent health & $\mathrm{X}$ & $\mathrm{X}$ & 2,013 & 3013 & 144 \\
\hline Aids and behavior & $\mathrm{X}$ & & 2,012 & 558 & 54 \\
\hline Aids patient care and stds & $\mathrm{X}$ & & 1,944 & 766 & 86 \\
\hline American journal of community psychology & $\mathrm{X}$ & & 1,922 & 1859 & 46 \\
\hline Psychology \& health & $\mathrm{X}$ & & 1,796 & 1196 & 49 \\
\hline Health education \& behavior & $\mathrm{X}$ & & 1,720 & 842 & 48 \\
\hline Accident analysis and prevention & $\mathrm{X}$ & & 1,717 & 2181 & 131 \\
\hline Aids education and prevention & $\mathrm{X}$ & & 1,700 & 1014 & 60 \\
\hline Aids care-psychological and socio-medical aspects of Aids/HIV & $\mathrm{X}$ & & 1,644 & 1510 & 114 \\
\hline Health risk \& society & $\mathrm{X}$ & & 1,634 & 186 & 25 \\
\hline Injury prevention & $\mathrm{X}$ & $\mathrm{X}$ & 1,536 & 840 & 69 \\
\hline Public health reports & $\mathrm{X}$ & $\mathrm{X}$ & 1,526 & 2469 & 94 \\
\hline Health \& place & $\mathrm{X}$ & & 1,492 & 437 & 29 \\
\hline Womens health issues & $\mathrm{X}$ & & 1,479 & 344 & 29 \\
\hline Health education research & $\mathrm{X}$ & & 1,303 & 1371 & 64 \\
\hline Journal of safety research & $\mathrm{X}$ & & 1,263 & 457 & 61 \\
\hline Journal of public health management and practice & $\mathrm{X}$ & & 1,235 & 815 & 0 \\
\hline Health promotion international & $\mathrm{X}$ & & 1,159 & 496 & 41 \\
\hline European journal of public health & $\mathrm{X}$ & $\mathrm{X}$ & 1,118 & 676 & 103 \\
\hline Journal of public health & $\mathrm{X}$ & $\mathrm{X}$ & 1,031 & 101 & 64 \\
\hline Journal of american college health & $\mathrm{X}$ & & 1,000 & 791 & 42 \\
\hline Public health & $\mathrm{X}$ & $\mathrm{X}$ & 0,986 & 808 & 155 \\
\hline Australian and New Zealand journal of public health & $\mathrm{X}$ & $\mathrm{X}$ & 0,976 & 970 & 85 \\
\hline $\begin{array}{l}\text { Canadian journal of public health-revue canadienne de sante } \\
\text { publique }\end{array}$ & $\mathrm{X}$ & & 0,976 & 1256 & 102 \\
\hline Community mental health journal & $\mathrm{X}$ & & 0,962 & 792 & 52 \\
\hline International journal of occupational and environmental health & $\mathrm{X}$ & $\mathrm{X}$ & 0,962 & 393 & 57 \\
\hline Health & $\mathrm{X}$ & & 0,935 & 155 & 23 \\
\hline Journal of public health policy & $\mathrm{X}$ & $\mathrm{X}$ & 0,930 & 255 & 20 \\
\hline Journal of womens health & $\mathrm{X}$ & $\mathrm{X}$ & 0,897 & 480 & 81 \\
\hline Journal of palliative care & $\mathrm{X}$ & & 0,875 & 569 & 31 \\
\hline Health \& social care in the community & $\mathrm{X}$ & & 0,869 & 384 & 54 \\
\hline Journal of rural health & $\mathrm{X}$ & $\mathrm{X}$ & 0,866 & 461 & 55 \\
\hline Rreproductive health matters & $\mathrm{X}$ & & 0,857 & 279 & 33 \\
\hline Studies in family planning & $\mathrm{X}$ & & 0,830 & 666 & 28 \\
\hline Journal of health care for the poor and underserved & $\mathrm{X}$ & & 0,815 & 382 & 79 \\
\hline Family \& community health & $\mathrm{X}$ & & 0,763 & 263 & 26 \\
\hline Journal of community psychology & $\mathrm{X}$ & & 0,732 & 806 & 45 \\
\hline Scandinavian journal of public health & $\mathrm{X}$ & $\mathrm{X}$ & 0,727 & 356 & 73 \\
\hline Journal of school health & $\mathrm{X}$ & & 0,721 & 828 & 54 \\
\hline Public health nursing & $\mathrm{X}$ & $\mathrm{X}$ & 0,693 & 479 & 61 \\
\hline Salud publica de México & $\mathrm{X}$ & & 0,689 & 481 & 49 \\
\hline Journal of community health & $\mathrm{X}$ & & 0,657 & 531 & 32 \\
\hline Scandinavian journal of caring sciences & $\mathrm{X}$ & & 0,650 & 433 & 57 \\
\hline American journal of health behavior & $\mathrm{X}$ & & 0,640 & 370 & 53 \\
\hline Administration and policy in mental health & $\mathrm{X}$ & & 0,556 & 219 & 25 \\
\hline Gesundheitswesen & $\mathrm{X}$ & & 0,548 & 730 & 125 \\
\hline
\end{tabular}


Tabla 2 (Cont.)

Revistas indizadas en la categoría Public, Environmental and Occupational Health del Social Sciences Citation Index*

\begin{tabular}{|l|c|c|c|c|c|}
\hline & SSCI & SCI & FI & $\begin{array}{c}\text { Total citas } \\
\mathbf{2 0 0 5}\end{array}$ & $\begin{array}{c}\text { Artículos } \\
\mathbf{2 0 0 5}\end{array}$ \\
\hline International journal of health planning and management & $\mathrm{X}$ & & 0,541 & 187 & 20 \\
\hline Childrens health care & $\mathrm{X}$ & & 0,487 & 151 & 20 \\
\hline Women \& health & $\mathrm{X}$ & & 0,431 & 795 & 27 \\
\hline Journal of the royal society for the promotion of health & $\mathrm{X}$ & & 0,381 & 97 & 11 \\
\hline Journal of religion \& health & $\mathrm{X}$ & & 0,298 & 113 & 25 \\
\hline Revista de saude publica & $\mathrm{X}$ & & 0,287 & 607 & 133 \\
\hline $\begin{array}{l}\text { Biosecurity and bioterrorism-biodefense strategy practice and } \\
\text { science }\end{array}$ & $\mathrm{X}$ & & & & \\
\hline Health expectations & $\mathrm{X}$ & $\mathrm{X}$ & & & \\
\hline International journal of occupational safety and ergonomics & $\mathrm{X}$ & & & & \\
\hline Journal of health and social behavior & $\mathrm{X}$ & & & & \\
\hline Journal of occupational health psychology & $\mathrm{X}$ & & & & \\
\hline Maternal and child health journal & $\mathrm{X}$ & & & & \\
\hline Prevention science & $\mathrm{X}$ & & & & \\
\hline Revista española de salud publica & $\mathrm{X}$ & & & & \\
\hline Revista panamericana de salud publica & $\mathrm{X}$ & & & & \\
\hline
\end{tabular}

* Ordenadas según factor de impacto de 2005.

Tabla 3

Países de publicación de las revistas indizadas en la categoría Public, Environmental and Occupational Health del Social Sciences Citation Index

\begin{tabular}{|l|r|r|}
\hline \multirow{2}{*}{ Países } & \multicolumn{2}{c|}{ Revistas } \\
\cline { 2 - 3 } & \multicolumn{1}{c|}{$\mathbf{N}$} & \multicolumn{1}{c|}{$\%$} \\
\hline EE.UU. & 32 & 45,7 \\
\hline Inglaterra & 28 & 40,0 \\
\hline Canadá & 2 & 2,9 \\
\hline Australia & 1 & 1,4 \\
\hline Holanda & 1 & 1,4 \\
\hline Alemania & 1 & 1,4 \\
\hline Noruega & 1 & 1,4 \\
\hline Polonia & 1 & 1,4 \\
\hline España & 1 & 1,4 \\
\hline Brasil & 1 & 1,4 \\
\hline México & 1 & 1,4 \\
\hline
\end{tabular}

En este sentido sabemos que las revistas de ciencias experimentales concitan mayor citación que las de ciencias sociales y humanas, así como las revistas de especialidades 
Tabla 4

Editoriales con más de dos revistas indizadas en la categoría Public, Environmental and Occupational Health del Social Sciences Citation Index

\begin{tabular}{|l|c|r|}
\hline \multirow{2}{*}{\multicolumn{1}{|c|}{ Editoriales }} & \multicolumn{2}{c|}{ Revistas } \\
\cline { 2 - 3 } & $\mathbf{N}$ & $\mathbf{1}$ \\
\hline Blackwell Publishing & 8 & 11,4 \\
\hline Elsevier Science & 8 & 11,4 \\
\hline Taylor \& Francis & 5 & 7,1 \\
\hline Kluwer Academic & 4 & 5,7 \\
\hline Sage Publications & 4 & 5,7 \\
\hline Oxford University Press & 4 & 5,7 \\
\hline Springer/Plenum Publishers & 4 & 5,7 \\
\hline Mary Ann Liebert Inc & 3 & 4,3 \\
\hline BMJ Publishing Group & 3 & 4,3 \\
\hline Lippincott Williams \& Wilkins & 2 & 2,9 \\
\hline John Wiley \& Sons Inc & 2 & 2,9 \\
\hline Total & 47 & 67,1 \\
\hline
\end{tabular}

básicas alcanzan niveles de citación más elevados que las revistas de orientación aplicada (especialidades clínicas). Por tanto, en principio el FI de una revista será comparable exclusivamente al de otras revistas clasificadas en la misma categoría. Así, por ejemplo, en la tabla 1 sólo son comparables entre sí los factores de impacto de las cuatro revistas incluidas en la categoría Pharmacology and Pharmacy. Y Medicina Clínica y la Revista Clínica Española, ya que están indizadas en la misma categoría del mismo índice. Y también lo son Neurología y Revista de Neurologie International microbiology y Enfermedades infecciosas y microbiología clínica. El resto de revistas de la tabla no son comparables entre sí por su FI, como no lo son con otras revistas que no estén en la misma categoría cualquiera que sea su nacionalidad. Sólo con determinadas cautelas y mediante transformaciones de los valores de los índices se pueden sal- var estas diferencias entre categorías para realizar comparaciones entre ellas.

3. El tipo de revista y, particularmente, el tipo de trabajos que publique condicionará su FI. Así, las revisiones, los artículos metodológicos, las cartas, los artículos polémicos, son trabajos con una alta e inmediata atención de los lectores, por lo que su potencialidad de citación es mayor. Esto quiere decir que una revista que publique habitualmente este tipo de trabajos puede alcanzar mejores niveles de citación.

4. El número de trabajos de la revista afecta al FI, de manera que las revistas que más trabajos publican al año muestran una menor variabilidad en sus impactos que las que publican pocos.

5. La fórmula utilizada para calcular el FI posee también unos efectos un tanto perversos. El FI es un cociente entre el número de 
citas (numerador) y el número de artículos publicados (denominador). Pues bien, mientras que el ISI en el numerador incluye las citas a todos los trabajos publicados por una revista (artículos, revisiones, resúmenes de congresos, editoriales...) en el denominador sólo incluye los artículos originales o de revisión. Esta fórmula favorece a aquellas revistas que publiquen muchos trabajos de carácter menor que no sean artículos o revisiones.

6. La rapidez en la publicación, e indirectamente la periodicidad de una revista, es un factor clave para conseguir mayores tasas de citación. Cuanto antes estén los artículos a disposición de los lectores antes podrán ser citados.

Teniendo en cuenta todas estas cautelas podrá hacerse una lectura adecuada del impacto que obtenga la RESP en el futuro. En cualquier caso, hay que felicitarse de que la RESP haya conseguido estar entre las revistas científicamente más influyentes del mundo, algo que se puede exhibir con orgullo. Es un logro de la revista, de todos los que la hacen posible día a día y, por encima de todo, de las personas que publican en ella sus trabajos, de las que los evalúan y de las que los leen.

\section{BIBLIOGRAFÍA}

1. Thomson. About us. Disponible en: http:// www.thomson.com/ (Consultado el 1 de julio de 2006).

2. Web of Science. Disponible en: http://www.accesowok.fecyt.es/login/(Consultado el 1 de julio de 2006).
3. Ruiz Pérez R, Delgado López-Cózar E, Jiménez Contreras E. Criterios del Institute for Scientific Information para la selección de revistas científicas. Su aplicación a las revistas españolas: metodología e indicadores. Int J Clin Health Psychol 2006, 6(2): 401-24.

4. Resolución de 25 de octubre de 2005, de la Presidencia de la Comisión Nacional Evaluadora de la Actividad Investigadora, por la que se establecen los criterios específicos en cada uno de los campos de evaluación. (BOE núm 266 de noviembre de 2005). Disponible en: http://www.mec.es/ciencia/ jsp/plantilla.jsp?area $=$ cneai $\&$ id $=501$

5. Resolución de 18 de febrero de 2005 de la Dirección General de Universidades por la que se modifican determinados aspectos de procedimiento de presentación de solicitudes de evaluación o informe de la ANECA para la contratación de personal docente e investigador, así como los criterios de evaluación, establecidos en las resoluciones de 17 de diciembre de 2002 y de 24 de junio de 2003, de dicha Dirección General. (BOE múm. 54 de 4 de marzo de 2005). Disponible en: http://www.aneca.es/

6. Garfield E. Citation Indexing. Its theory and applications in Science, Technology and Humanities. New York: Wiley; 1979.

7. Garfield E. Current Content 1994; (25): 3-7

8. Garfield E. The Agony and the Ecstasy. The History and Meaning of the Journal Impact Factor. International Congress on Peer Review And Biomedical Publication. Chicago, 16 de Septiembre de 2005. Disponible en http://garfield.library.upenn. edu/papers/jifchicago2005.pdf

9. Seglen PO. Why the impact factor of journals should not be used for evaluating research. BMJ 1997; 314: 498-502.

10. Amin M, Mabe M. Impact Factors: Use and abuse. Perspectives in Publishing 2000, 1: 1-6. 